\title{
Validation of the Lockheed Martin Morphing Concept with Wind Tunnel Testing
}

\author{
Thomas G. Ivanco ${ }^{1}$ and Robert C. Scott ${ }^{2}$ \\ NASA Langley Research Center, Hampton VA, 23681 \\ Michael H. Love ${ }^{3}$ and Scott Zink ${ }^{4}$ \\ Lockheed Martin Aeronautics Company, Fort Worth, TX, 76108 \\ and \\ Terrence A. Weisshaar ${ }^{5}$ \\ Purdue University, West Lafayette, IN, 47907
}

\begin{abstract}
The Morphing Aircraft Structures (MAS) program is a Defense Advanced Research Projects Agency (DARPA) led effort to develop morphing flight vehicles capable of radical shape change in flight. Two performance parameters of interest are loiter time and dash speed as these define the persistence and responsiveness of an aircraft. The geometrical characteristics that optimize loiter time and dash speed require different geometrical planforms. Therefore, radical shape change usually involving wing area and sweep - allows vehicle optimization across many flight regimes. The second phase of the MAS program consisted of wind tunnel tests conducted at the NASA Langley Transonic Dynamics Tunnel to demonstrate two morphing concepts and their enabling technologies with large-scale semi-span models. This paper will focus upon one of those wind tunnel tests that utilized a model developed by Lockheed Martin Aeronautics Company (LM). Wind tunnel success criteria were developed by NASA to support the DARPA program objectives. The primary focus of this paper will be the demonstration of the DARPA objectives by systematic evaluation of the wind tunnel model performance relative to the defined success criteria. This paper will also provide a description of the LM model and instrumentation, and document pertinent lessons learned. Finally, as part of the success criteria, aeroelastic characteristics of the LM derived MAS vehicle are also addressed. Evaluation of aeroelastic characteristics is the most detailed criterion investigated in this paper. While no aeroelastic instabilities were encountered as a direct result of the morphing design or components, several interesting and unexpected aeroelastic phenomenon arose during testing.
\end{abstract}

\section{Introduction}

$\mathrm{P}$ HASE II of the Defense Advanced Research Projects Agency (DARPA) Morphing Aircraft Structures (MAS) program is an effort to develop and evaluate morphing concepts in a wind tunnel environment to enable technologies for a future unmanned flight demonstrator program. As part of the DARPA Phase II program, contracts were awarded to Lockheed Martin Aeronautics Company (LM) and NextGen Aeronautics Inc., for the design, fabrication, and testing of a wind tunnel MAS model. This paper will focus upon the LM variant of the MAS Phase II wind tunnel programs.

Four entities were involved in the phase II testing of the LM MAS vehicle: DARPA, Lockheed Martin, the Air Force Research Laboratory (AFRL), and NASA. DARPA initiated the MAS program, funded the research and testing, and guided the program by directing the test objectives. Lockheed Martin was the contractor tasked to design, fabricate, test, and analyze an operational MAS wind tunnel model. The role of AFRL was to serve as a program technical monitor and advisor to DARPA. The primary role of NASA was to supply and operate the wind

\footnotetext{
${ }^{1}$ Aerospace Engineer, Aeroelasticity Branch, MS 340, AIAA Member.

${ }^{2}$ Senior Aerospace Engineer, Aeroelasticity Branch, MS 340, AIAA Associate Fellow.

${ }^{3}$ Senior Staff Engineer, AIAA Associate Fellow.

${ }^{4}$ Senior Engineer, AIAA Associate Fellow.

${ }^{5}$ Professor, Former DARPA Program Manager, School of Aeronautics and Astronautics, AIAA Fellow.
} 
tunnel facility. Additionally, NASA acted as an agent to DARPA evaluating the model design process, assisting in the development of the test plan, and evaluating the success of the wind tunnel test. As a result, NASA developed measurable wind tunnel success criteria to support the DARPA directed program objectives.

This paper is divided into four sections: definition of program objectives and test success criteria, model and wind tunnel description, results that satisfy the wind tunnel test success criteria, and lessons learned. Included in the model description section are the identified critical enabling technologies. The most detailed item of the test success criteria section is an identification of the aeroelastic characteristics of the morphing concept. Included in this section is a background of the analysis, a comparison of the theoretical flutter boundary and experimental encounters, a discussion about the effects of morphing motion on dynamic vehicle response, and details regarding observed dynamics of the flexible skin.

Lockheed Martin researchers are writing a parallel paper for presentation at this conference providing more information about the model design, fabrication, and validation of their analytical design tools. ${ }^{1}$ Detailed comparisons of analytical and experimental pressure and structural load data is provided in reference 1 in addition to correlation of the model with an envisioned flight demonstrator vehicle. Similarly, DARPA representatives are authoring a paper detailing the MAS program and the significance of the Phase II results. ${ }^{2}$

\section{Definition of Program Objectives and Test Success Criteria}

\section{A. Program Objectives}

The MAS Phase II wind tunnel test program objectives, as identified by DARPA, were:

1) Demonstrate morphing wing operability under realistic aerodynamic loads;

2) Obtain test data to validate analytical prediction methods for morphing aircraft;

3) Minimize the departure of the wind tunnel model design and test conditions from those of the envisioned flight demonstrator aircraft.

\section{B. Test Success Criteria}

In order to achieve these program objectives, Lockheed Martin was tasked to design a wind tunnel model that allows for a radical shape change under aerodynamic loads representative of a steady state $1 \mathrm{~g}$ flight condition and to capture data for the validation of analytical prediction methods. The use of actual flight hardware was encouraged. Considering these aspects of the program objectives, the following seven wind tunnel success criteria were identified by NASA and endorsed by DARPA:

1) Demonstrate actuation of the morphing structure with approximately $1 \mathrm{~g}$ of aerodynamic load, without structural failure or binding;

[1g was determined from the envisioned flight vehicle gross weight and then scaled to match the dimensions of the wind tunnel model. At the TDT model scale, this equates to a full-span vehicle gross weight of $5400 \mathrm{lbs}$, and a semi-span model load of $2700 \mathrm{lbs}$.]

2) Demonstrate model integrity and stability for varying configurations throughout a Mach range of 0.2 to 0.9 and representative altitudes from 10,000 to 50,000 feet;

[Facility capabilities, combined with the envisioned flight envelope, dictated the wind tunnel test matrix.]

3) Identify aeroelastic characteristics associated with morphing structures and transition under air loads;

[One of the unknowns prior to testing was the effect of the flexible morphing components and wing motion on the aeroelastic characteristics of the model. The complex structure needs to demonstrate adequate static stiffness. Also, the wing motion associated with morphing transition and the actuator systems must not induce an instability.]

4) Demonstrate flexible skins and the resilience of those skins to air loads and actuation;

[Morphing concepts often require large amounts of flexible skin on the wing surface. Significant and uncontrolled skin deformation may comprise the aerodynamic shape and alter vehicle performance. This criterion was dictated to demonstrate the ability to withstand high velocity airflows and lift generated suction without significant skin deformation.]

5) Demonstrate successful use of flight representative hardware to operate the actuation systems; 
[This and the following two criteria were specified as measurable enabling features required for the development of a future flight demonstrator vehicle.]

6) Demonstrate successful mating of the morphing structures and seamless gap closures;

7) Acquire specified sets of wind tunnel data to correlate with design analysis.

\section{Model and Wind Tunnel Description}

\section{A. General Model Characteristics}

Lockheed Martin developed a double-hinged folding-wing concept of a flying-wing vehicle. The wind tunnel model was a semi-span half-scale representation of this vehicle concept. A multiple-exposure photograph of the model mounted in the wind tunnel is shown in figure 1. Figure 1 captures the morphing motion at different stages of actuation from a loiter configuration, in which the wing is fully extended, to the dash configuration, in which the wing is fully folded. To operate the wing fold system, the wind tunnel model used two electrically slaved motors and a drive train system. In order to close the gap between the inboard wing and fuselage in the dash configuration, a thermopolymer actuator was developed to drive a leading edge flap. Flexible skin material was utilized across the wing fold and flap regions. The inclusion of these skins was necessary to maintain a clean outer mold line while still permitting radical wing shape change. To minimize skin deformation, a vacuum system was developed to maintain suction on the seamless skins and hold them against the wing internal structure.

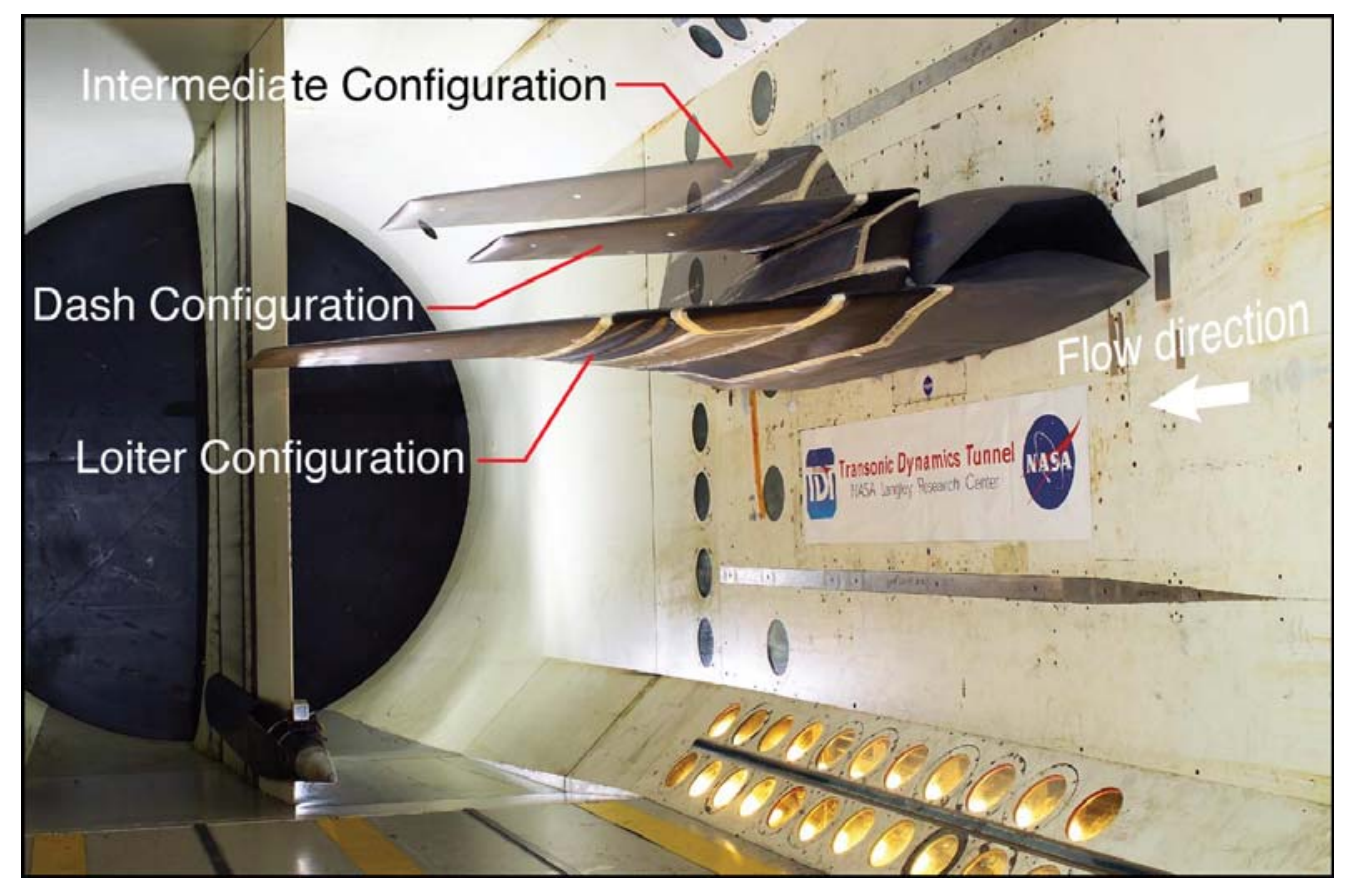

Figure 1. Multiple Exposure Photograph of Lockheed Martin MAS Wind Tunnel Model During a Morphing Sequence

Lockheed Martin is writing a parallel conference paper which presents significantly more details about the model design, components, and fabrication. ${ }^{1}$ To summarize, the semi-span wind tunnel model dimensions were ${ }^{1,3}$ :

- $\quad$ Wing area (loiter) $=62.5 \mathrm{ft}^{2}$

- $\quad$ Span (loiter) $=9.3 \mathrm{ft}$

- $\quad$ Length $=13.6 \mathrm{ft}$

- Model weight $~ 1700 \mathrm{lbs}$

By actuating the wing fold system from the dash to the loiter position, the model experienced the following planform changes ${ }^{1,3}$ :

- $2.8 x$ wing area increase

- $1.7 x$ span increase 
- $1.3 x$ increase in wetted area

- 30 deg wing sweep change of the $1 / 2$ chord

This large model dimension was driven by the DARPA requirement to use actuation and structural concepts representative of a future flight demonstrator. Unfortunately, this large size resulted in unanticipated coupling with the wind tunnel support system flexibility. Also, because of its size, the model extended outside of the region of the test section in which flow conditions are constant, making it more difficult to validate CFD predictions.

Critical enabling technologies for satisfying the wind tunnel success criteria and preparing for a future flight demonstrator vehicle were identified by Lockheed Martin and briefly described here.

\section{Seamless Skin Material}

Two skin materials were investigated: re-enforced silicone elastomeric for use in the wind tunnel model, and shape memory polymers envisioned for the flight vehicle. The shape memory polymer material was not adequately developed within the schedule allotted for the wind tunnel model entry. Further development will result in a stiffer material and a better airfoil outer mold line across the wing fold areas. Also, shape memory material may eliminate the need for a vacuum otherwise required to stabilize the silicone elastomeric material when exposed to airflow.

\section{Seamless Skin Support Structure}

Due to the flexibility of the seamless skins, a complex substructure was developed in the wing fold regions housing wing structure, actuation components, instrumentation, and vacuum tubes. This structure also had to provide the basis for an airfoil shape and allow for 130 degrees of wing fold while withstanding load deformation. Perhaps a preferred technology would be the development of a more rigid skin eliminating the need for a substructure to support it under aerodynamic loads. This would also allow for better distribution of load through the fold regions and possibly a reduction in structural weight.

\section{Thermopolymer Actuators}

For the leading edge flap actuator, an increase in reliability and a reduction in weight, complexity, and operating time of the thermopolymer actuator are required for application to a flight vehicle. Also, further development of the envisioned wing fold actuators is needed if desired for flight vehicle use.

\section{Wing Fold Control}

In order to reliably change shape in a flight vehicle, precise, repeatable, stable, and symmetrical wing actuation is required. The wind tunnel model accomplished morphing actuation using two commercial off-the-shelf electrically slaved drive motors with a position feedback control system developed by Minarik Automation and Control.

\section{B. Model Instrumentation and Data Acquisition}

The wind tunnel model was mounted to a five component strain gauge balance that measured normal and axial forces, and pitch, roll, and yaw moments. Connected to the balance was a standard TDT supporting strut which is driven in rotation about the pitch axis to control angle of attack. Lift and drag were calculated from the balance force components and corrected for model angle of attack. Model angle of attack was measured with a Q-flex accelerometer mounted in the fuselage near the balance. Wing fold angles were measured with potentiometers, and the wing fold drive train position was measured with drive motor position counters. Additional instrumentation included 37 steady pressure taps, 14 high response unsteady pressure transducers, eight accelerometers, and strain gauges. Strain gauge measurements were used to calculate wing-fold-line hinge moments at seven different locations and axial load on the leading edge flap actuator rod.

Data was sampled at a rate of $500 \mathrm{~Hz}$ and low-pass filtered at $200 \mathrm{~Hz}$. Nominal data samples were five seconds in length and morphing sequences were extended to 75 second records.

\section{Wind Tunnel Facility}

Wind tunnel testing was conducted in the NASA Langley Transonic Dynamics Tunnel (TDT) because of the facility's large test section, Mach number range, and variable pressure capabilities. Also, the TDT is a dedicated aeroelastic test facility designed for high risk dynamic testing and has built-in safety features such as a rapid flow speed reduction capability, observation windows, and a model catch screen. The TDT has a 16 x 16 feet test section and can produce continuous flow speeds from near zero to a Mach number of 1.2 in either air or R134a heavy gas. Total pressure within the tunnel can be varied from a near vacuum to sea level. Maximum Reynolds number is 
nearly 10 million per foot in heavy gas. Reference 4 describes the wind tunnel in detail and includes operational envelopes and tunnel characteristics.

\section{Results}

The results from the test and analysis of the LM MAS model are presented in the context of the seven success criteria enumerated earlier in this paper.

\section{A. Success Criterion 1: Demonstrate actuation of morphing structure with approximately $1 \mathrm{~g}$ of aerodynamic load, without structural failure or binding.}

Figure 2 shows a 75 second data record acquired during a morphing sequence under a constant $1 \mathrm{~g}$ aerodynamic load. Presented in figure 2 are the wing fold angles, model angle of attack (AOA), lift, and inboard and outboard motor torques.
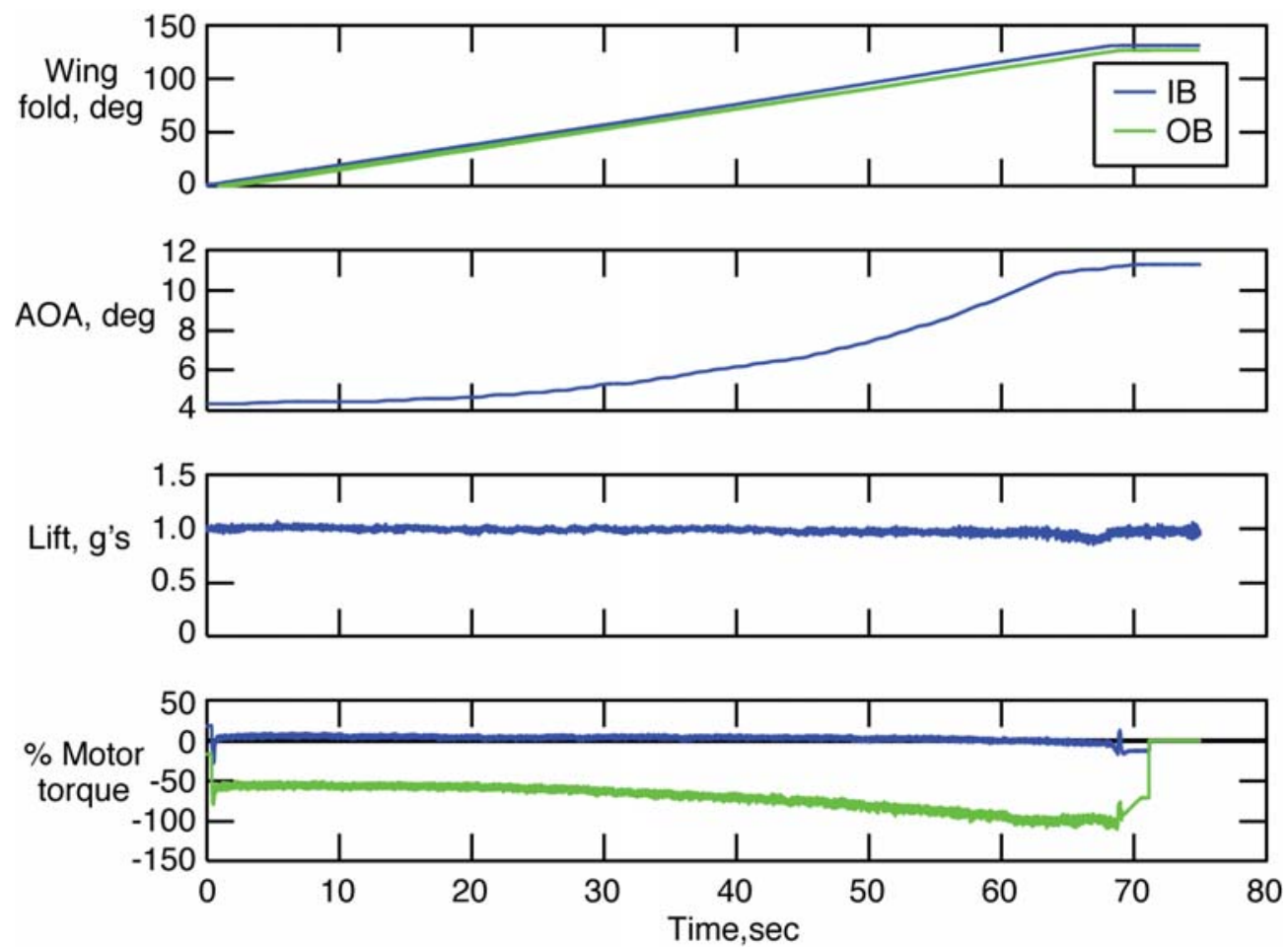

Figure 2. Constant 1g Morphing Sequence at Mach 0.6

During this actuation sequence, the wing was folded from the loiter ( 0 deg wing fold) to the dash (130 deg wing fold) position. The motor torque presented is in percent of the maximum continuous rated torque for each drive motor. One can see that the inboard motor is under very little load since the aerodynamic loads act in the direction of wing motion until the final phases of folding. The outboard motor torque, however, is much larger since it is moving in a direction opposing the aerodynamic load. The sign of the motor torque indicates the direction that the motor torque is being applied.

Also shown is the AOA which was manually controlled in an attempt to maintain a constant lift. The wandering of the mean lift value was caused by an inexact manual control of AOA. The significant increase in AOA required to maintain a constant lift is an indicator of the drastic wing area change achieved by this vehicle. With the wing in the loiter configuration, an AOA of approximately 4.3 degrees generated $1 \mathrm{~g}$ of lift. By comparison, the wing in the dash configuration required an AOA of 11.2 degrees to produce the same lift.

Unplanned asymmetric wing morphing would be a catastrophic occurrence on an actual flight vehicle. In order to project the ability to morph in controlled flight with a semi-span model demonstration, one must analyze the precision and repeatability of the wing actuation. These data show smooth wing operation without ratcheting, binding, or controller feedback instabilities. Equally important is that the same wing fold drive train position was achieved for a given command position regardless of aerodynamic load. 
Unfolding the wing from the dash to loiter configuration presented a unique problem under high aerodynamic loads. Motor current from the more critically loaded outboard motor was fed back into the control system to monitor drive motor limits. During an unfolding sequence, the aerodynamic loads acted in the direction of outboard wing motion. At $1 \mathrm{~g}$ lift conditions, the outboard motor became a generator at wing fold angles between 100 to 120 degrees. As a result, the controller detected a motor current error and halted the wing motion. For lift conditions less than $1 \mathrm{~g}$, this current feedback was not an issue during the unfolding sequence. Also, morphing in the opposite direction, where the motion of the outboard wing opposed the aerodynamic loads, did not cause an error. Minarik, the developer of the wing fold controller, had identified a solution by implementing a resistor into the control system. However, it was determined that this modification was unnecessary since the morphing concept had already met the test objectives.

A series of three $1 \mathrm{~g}$ unfolding data sequences were taken with the wing motion restarted after resetting the controller error. A compilation of these data sequences is shown in figure 3.
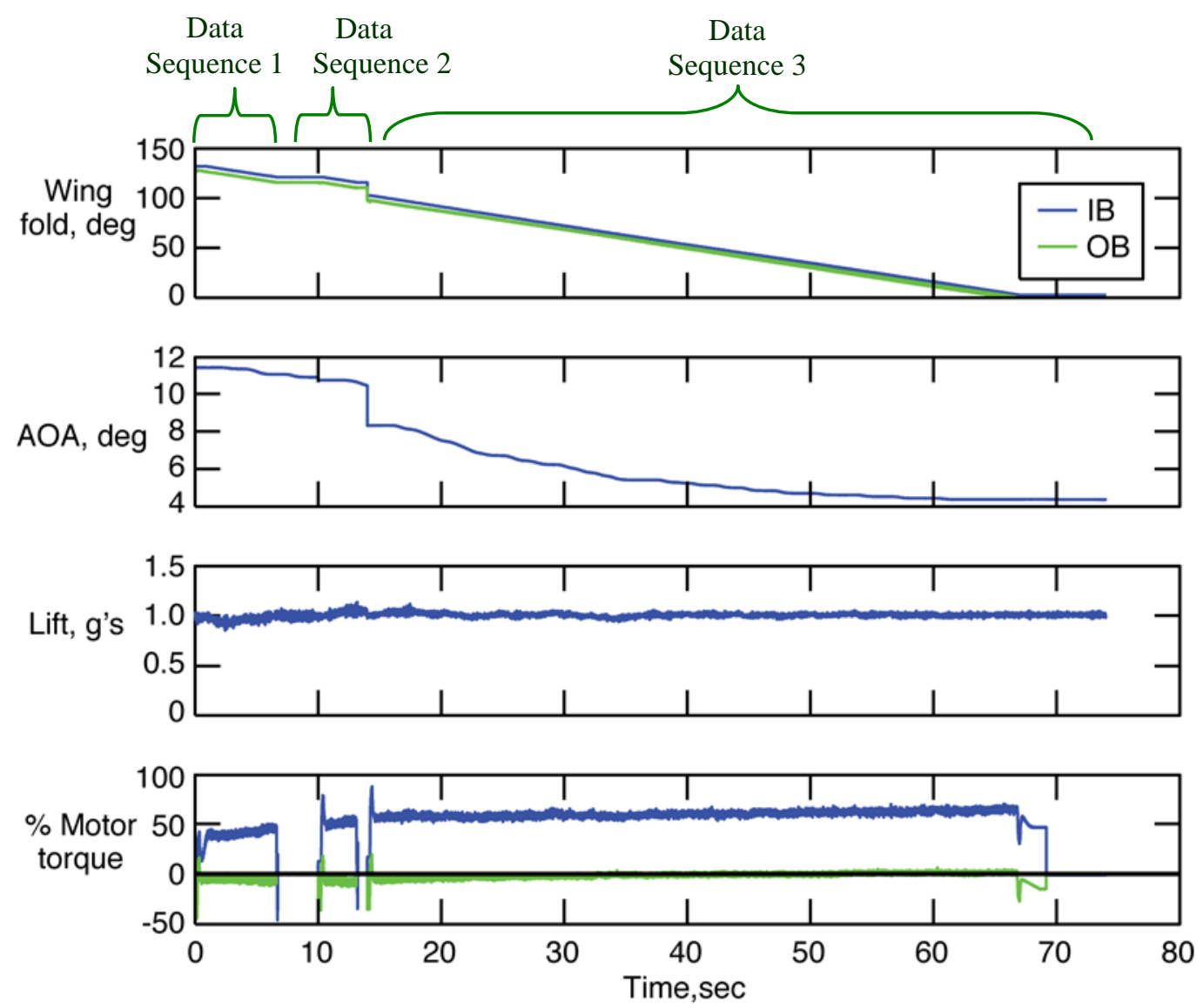

Figure 3. Constant 1g Morphing Sequence at Mach 0.6

For comparison, a smooth actuation sequence from the Dash to Loiter configuration is shown in figure 4 for a load of approximately $0.7 \mathrm{~g}$. 

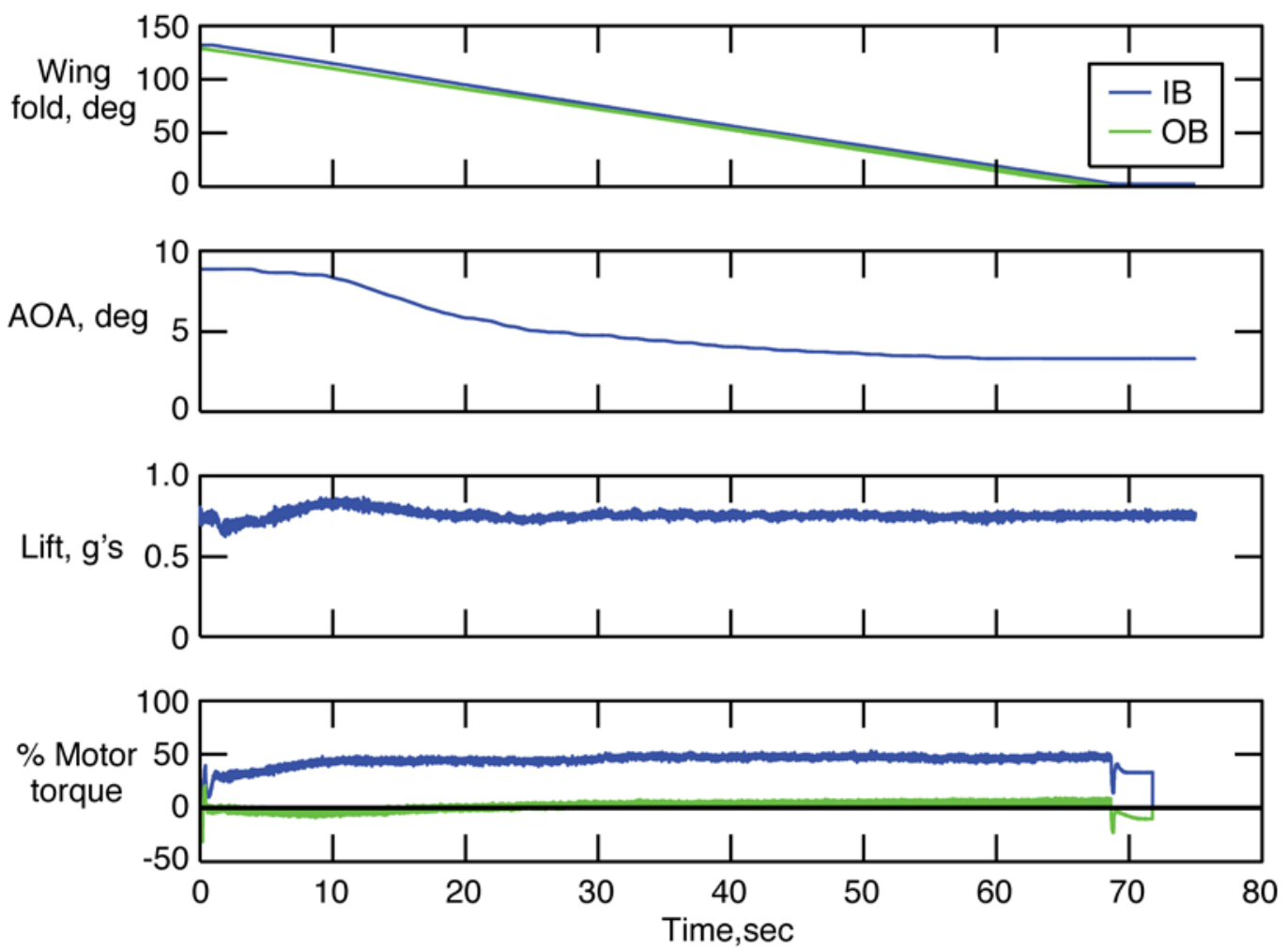

Figure 4. Constant 0.7g Morphing Sequence at Mach 0.6

\section{B. Success Criterion 2: Demonstrate model integrity and stability for varying configurations throughout a Mach range of 0.2 to 0.9 and representative altitudes from 10,000 to 50,000 feet.}

The test conditions varied from Mach numbers of 0.2 to 0.9 , and total pressures corresponding to altitudes from 8,000 to 70,000 feet. Not all configurations were tested at all conditions. Most morphing sequences were conducted at 0.6 Mach number and a representative altitude of approximately 30,000 feet.

In addition to morphing the wing and maintaining a constant lift condition, angle of attack sweeps were performed for many static wing fold angles and tunnel conditions. At several wind tunnel conditions, the model was taken beyond stall to a max angle of attack of 17 degrees in order to develop high angle data sets. Also, the static aerodynamic load was carried out to a maximum of approximately $1.5 \mathrm{~g}$ to verify structural integrity of the actuation system. Integrity was demonstrated by the drive system's ability to maintain position under loads in excess of $1 \mathrm{~g}$ without slippage. Shown in figure 5 is measured wing fold angle as a function of lift for three different nominal wing fold angles. The departures from nominal are indicative of the elastic deformation of the supporting structure. These elastic deflections are indicative of the flexibility expected in a flight vehicle, and therefore demonstrate the objective of providing a wind tunnel model with structures representative of a flight vehicle in the presence of morphing. During all of these wind tunnel conditions, the drive train system maintained a fixed commanded position. 

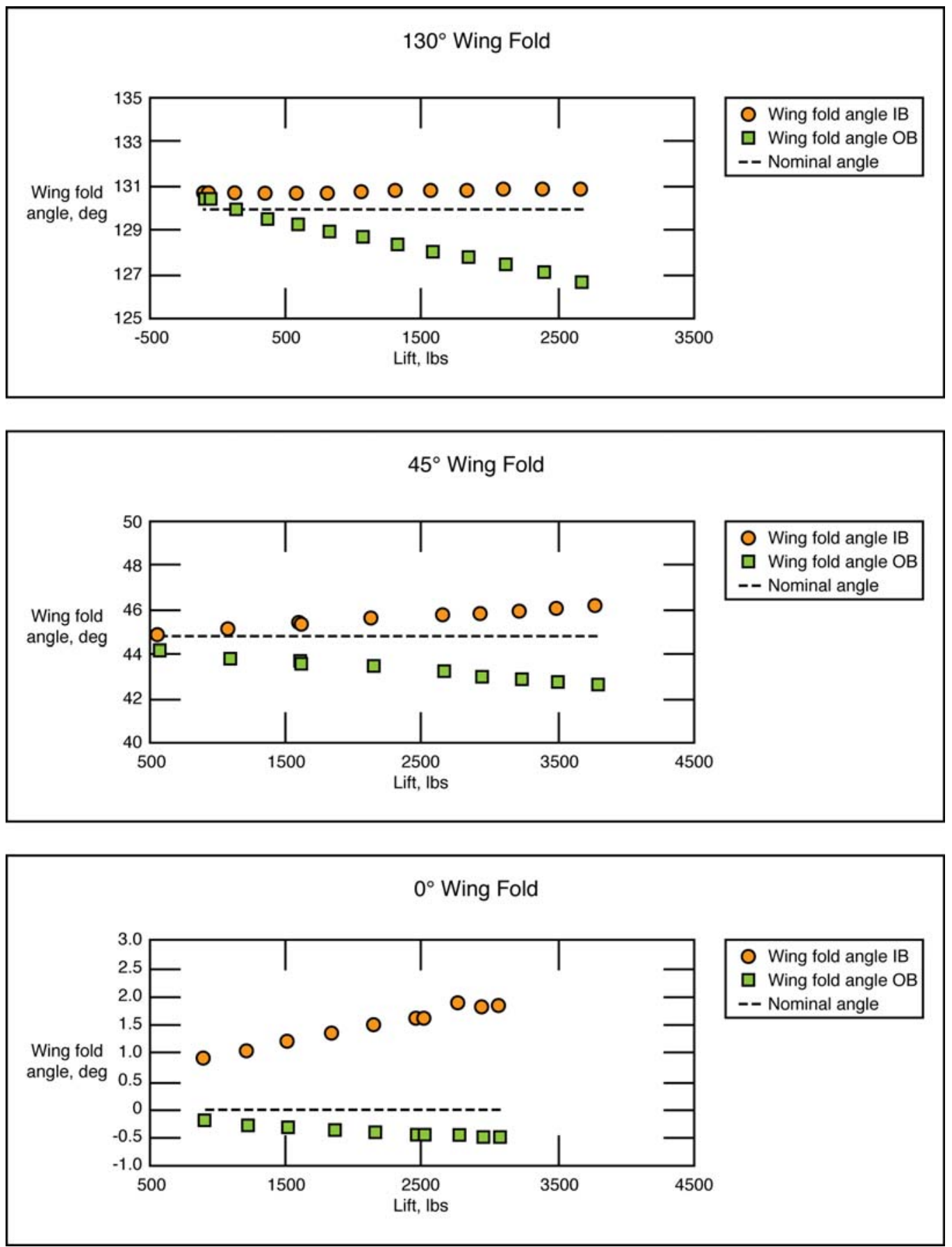

Figure 5. Measured Wing Fold Angles at Varying Load Conditions, Mach=0.6.

C. Success Criterion 3: Identify aeroelastic characteristics associated with morphing structures and transition under air loads.

\section{Background}

Flutter and divergence analyses were conducted by Lockheed Martin using NASTRAN finite element models coupled with doublet lattice aerodynamics. Validation of the flutter prediction methods was not an intentional goal of the wind tunnel test. Original LM predictions showed flutter dynamic pressures in the thousands of psf for all 
intended Mach numbers. The expected wind tunnel test boundary was limited to 200 psf in dynamic pressure. Therefore, flutter was considered a non-issue throughout model design and fabrication.

After balance selection, LM updated the flutter analysis to include balance flexibility. The results showed that the predicted flutter boundary dropped to approximately $550 \mathrm{psf}$ for the 65 deg wing fold configuration. These flutter analyses, with a soft-in-pitch balance, showed significant coupling between pitch and wing bending modeshapes of the model. The predicted flutter mode was a pitch motion of the model about the balance. Additional analyses with parameter variations revealed a significant sensitivity to pitch stiffness. Concern then arose regarding the stiffness of the TDT supporting strut that connects the model to the wind tunnel wall.

An initial estimate of the stiffness of the TDT supporting strut was conducted by NASA. This initial estimate showed the pitch stiffness of the supporting strut to be orders of magnitude greater than the balance. Therefore, the flutter analysis of the model and balance on a rigid backstop was considered to be an accurate prediction.

A detailed Ground Vibration Test (GVT) was conducted of the model with the flexible balance integrated and the system mounted on a nearly rigid backstop. Modes and frequencies correlated well the structural finite element model. Upon installing the model in the tunnel, however, it was discovered through an abbreviated GVT that the supporting strut was considerably more flexible in pitch than anticipated. (Details regarding the overestimated strut stiffness, and post-test flutter analyses using updated stiffness are provided in the lessons learned section.)

Since the 65 deg wing fold configuration produced the lowest flutter dynamic pressure in previous analyses, new flutter analyses were conducted on this configuration with the reduced stiffness. While this flutter analysis was being done, wind tunnel testing began with configurations thought to be less flutter critical, specifically the zero degree wing fold configuration. Unexpectedly, flutter was encountered with this configuration. After experimentally reaching the flutter condition, LM analytically determined that the critical flutter configuration with the more flexible strut was indeed zero degrees wing fold.

Figure 6 shows the analytical flutter boundaries for a rigid backstop and the updated boundaries with the flexible supporting strut for Mach numbers 0.6 and 0.8 .

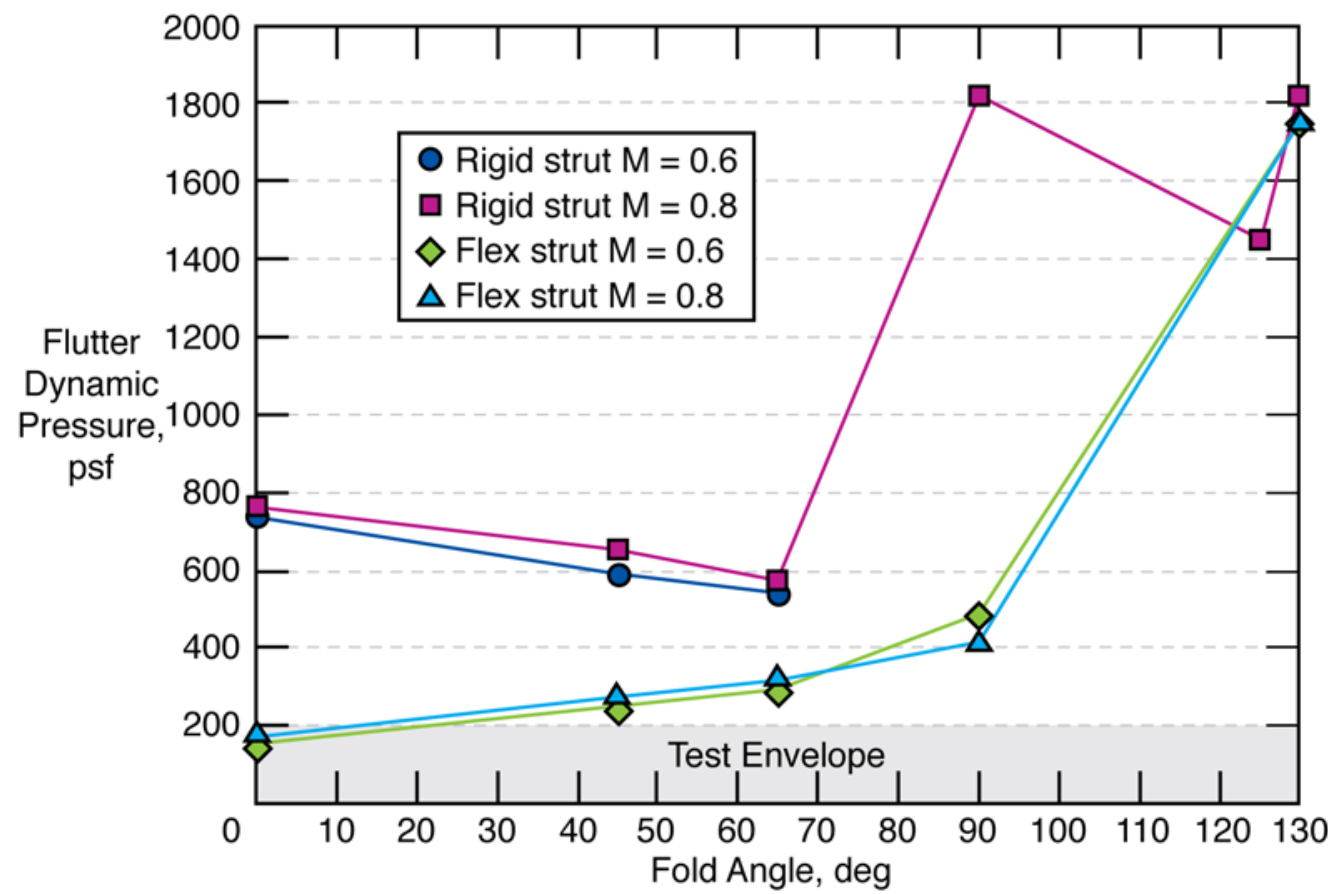

Figure 6. Analytical Flutter Boundaries with Flexible Balance.

\section{Comparison of Analytical and Experimental Flutter Results}

Correlations of the predicted flutter dynamic pressures, with the flexible strut modeled, and experimental trends at a Mach number of 0.6 were good. Although an experimental flutter condition was not experienced at a Mach number of 0.6 , numerous test conditions that were close to the predicted flutter boundary produced high dynamic responses that were indicative of a pending flutter condition. To avoid model and facility damage, testing was halted short of an actual flutter encounter. Additionally, the dynamic response of the model was greatly reduced at 
high wing fold angles commensurate with the prediction trends. Test experience and observed model behavior provide confidence in the flutter prediction values at a Mach number of 0.6 .

Analytical flutter predictions for a Mach number of 0.8 were not as accurate as the 0.6 Mach number results and were non-conservative by comparison to the experimental results. Understandably, the flutter condition is not expected to be predicted well with doublet lattice aerodynamics for a thick airfoil at high transonic speeds where viscous effects become important. An experimental flutter condition was encountered at a dynamic pressure of 138 psf for the zero degree wing fold configuration, compared to a value of 153 psf predicted in the analysis.

For the 45 deg wing fold configuration and a Mach number of 0.8 , however, flutter experimentally occurred significantly below the predicted dynamic pressure value shown in figure 6 . As shown in figure 6 , the analysis predicts a flutter dynamic pressure of approximately 275 psf, whereas the model experimentally encountered flutter at approximately 155 psf.

After further investigation, LM discovered that the critical flutter mode for wing fold angles in the vicinity of 45 and 65 degrees is a different mechanism than the zero degree fold angle. In these intermediate fold configurations, a mode with fore-aft motion on the outboard panel couples with a torsion motion of the inboard wing and a full-body pitch/yaw motion about the balance and supporting strut, as illustrated by figure 7 , and becomes the critical flutter mode shape. A v-g analysis shows this second flutter mode to be a "hump mode" characterized by a shallow crossing and a shallow return over a small range of velocities. The value of damping for this mode was near zero over the entire range of velocities and the crossing was initially overlooked during the flutter boundary analysis.

Both analytical flutter modes are strong functions of wing fold angle. Figure 8 shows these modes identified as "analysis mode 1" and "analysis mode 2" vs. fold angle. Predicted flutter dynamic pressure at 0.8 Mach number and 45 degrees of wing fold is approximately $170 \mathrm{psf}$ for the second mode, which is significantly closer to the experimental result. Figure 8 also includes the experimental flutter conditions encountered at a Mach number of 0.8.
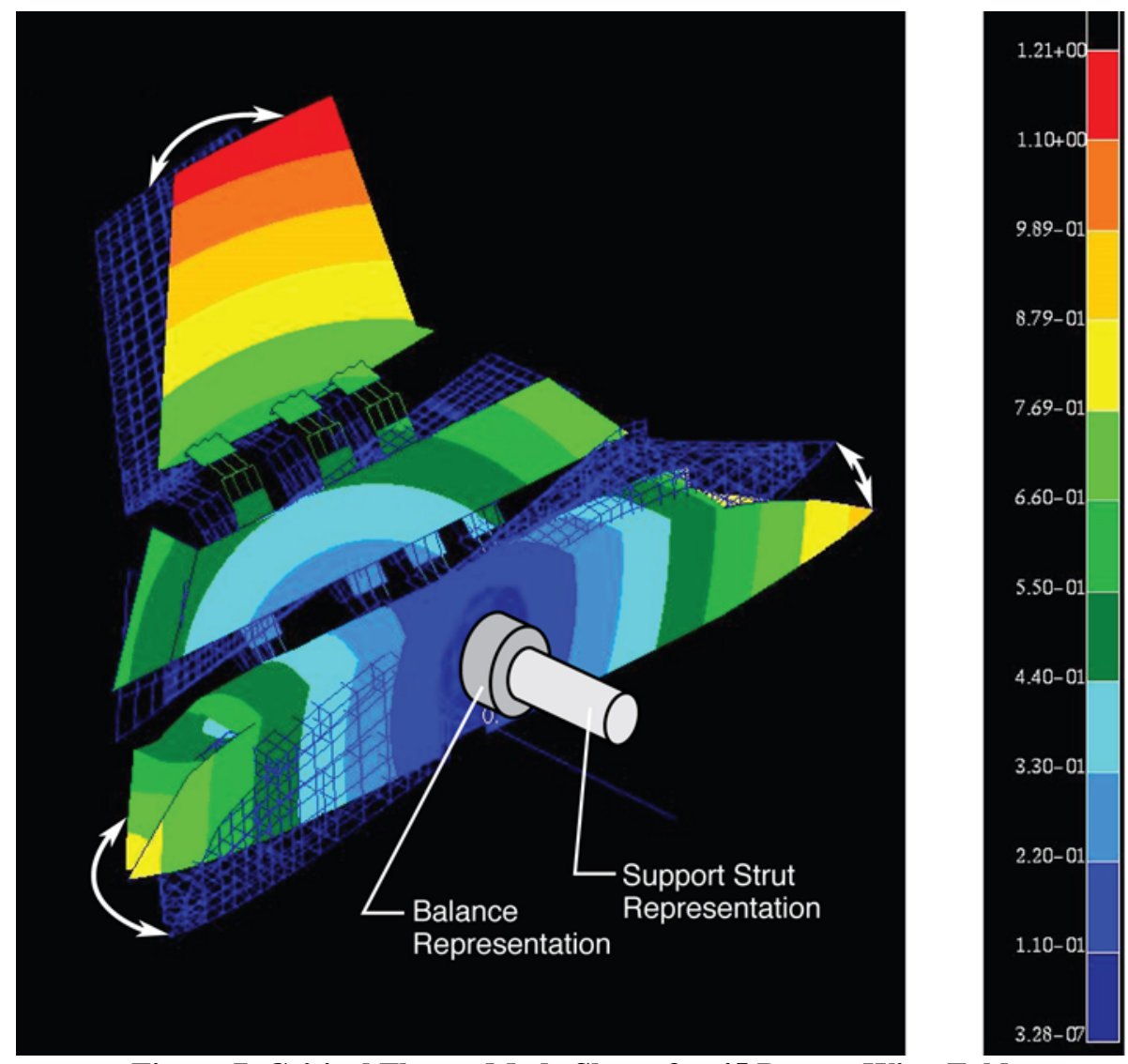

Figure 7. Critical Flutter Mode Shape for 45 Degree Wing Fold 


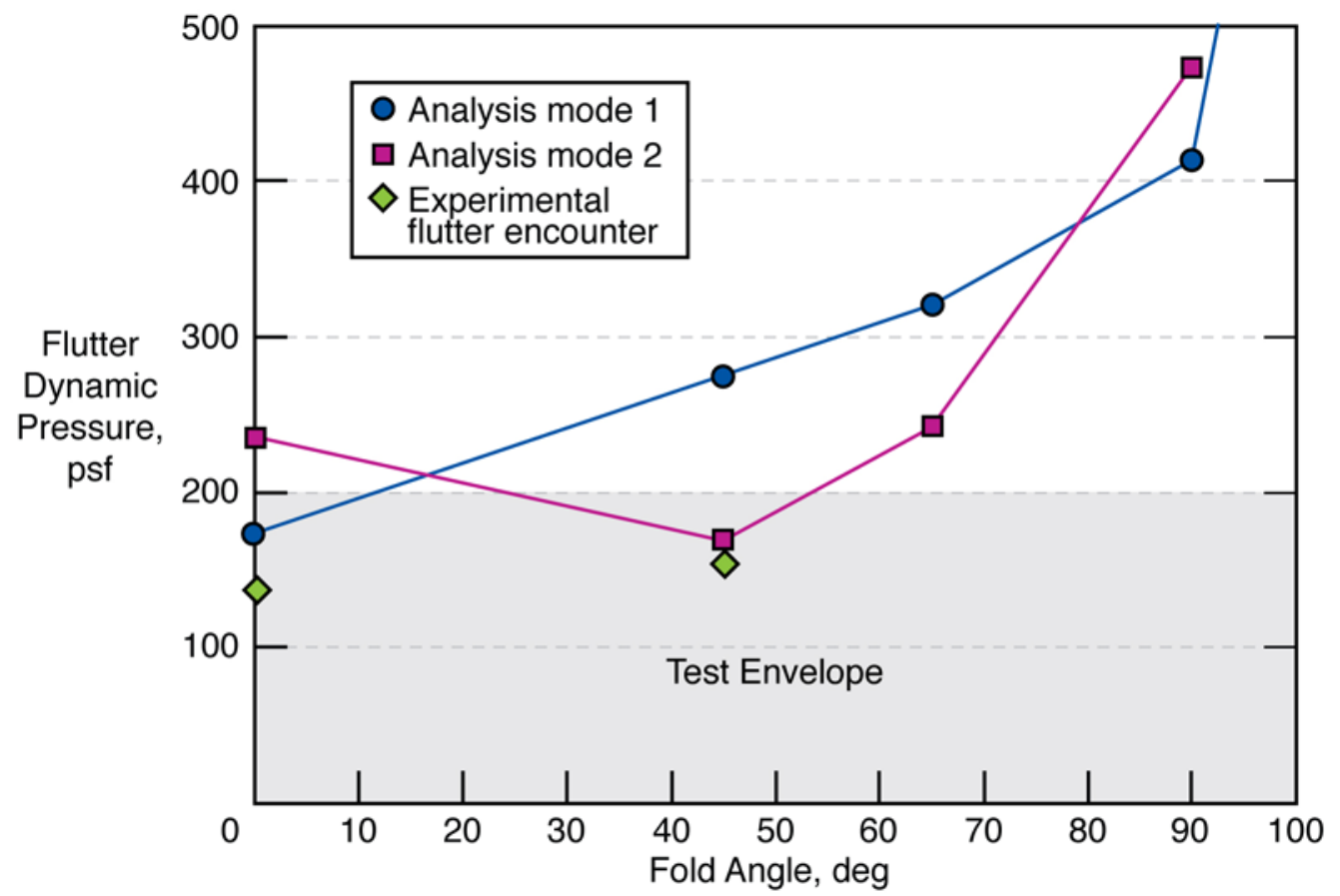

Figure 8. Flutter Dynamic Pressure as a Function of Wing Fold Angle. Flexible Strut. Mach $=0.8$

\section{Dynamic Response During Morphing Sequences}

Wing morphing operation was conducted near the expected flutter boundary at a Mach number of 0.6. As the wing moved through all fold positions, the dynamic response of the wing while moving was similar to the dynamic response of the wing at each fold position while stationary. Therefore, no additional dynamics or instabilities were attributed to the motion of the wing or the actuation components.

\section{Success Criterion 4: Demonstrate flexible skins and the resilience of those skins to air loads and actuation.}

Figure 9 is an underside view of the model in the loiter configuration. Easily visible in this photograph are the flexible seamless skins positioned along the wing fold hinge lines and the leading edge flap. Throughout testing, the skins proved to be generally robust and also easily repairable when tears occurred around a stress concentration area. Additionally, the wind tunnel test exposed the flexible skins to transonic flow and lift loads in excess of 1g. Cavity vacuum pressures were recorded at each test condition, and minimum required pressures were determined. During almost all of the test conditions, the flexible skins demonstrated resilience to the air loads, maintained the desired shape, and did not exhibit any "flag waving" or other visible dynamics. There were three conditions out of the thousands tested, however, where significant skin motion was observed by the test team. Although these occurrences of skin dynamics were rare, the characteristics are interesting and are therefore discussed in detail below. 


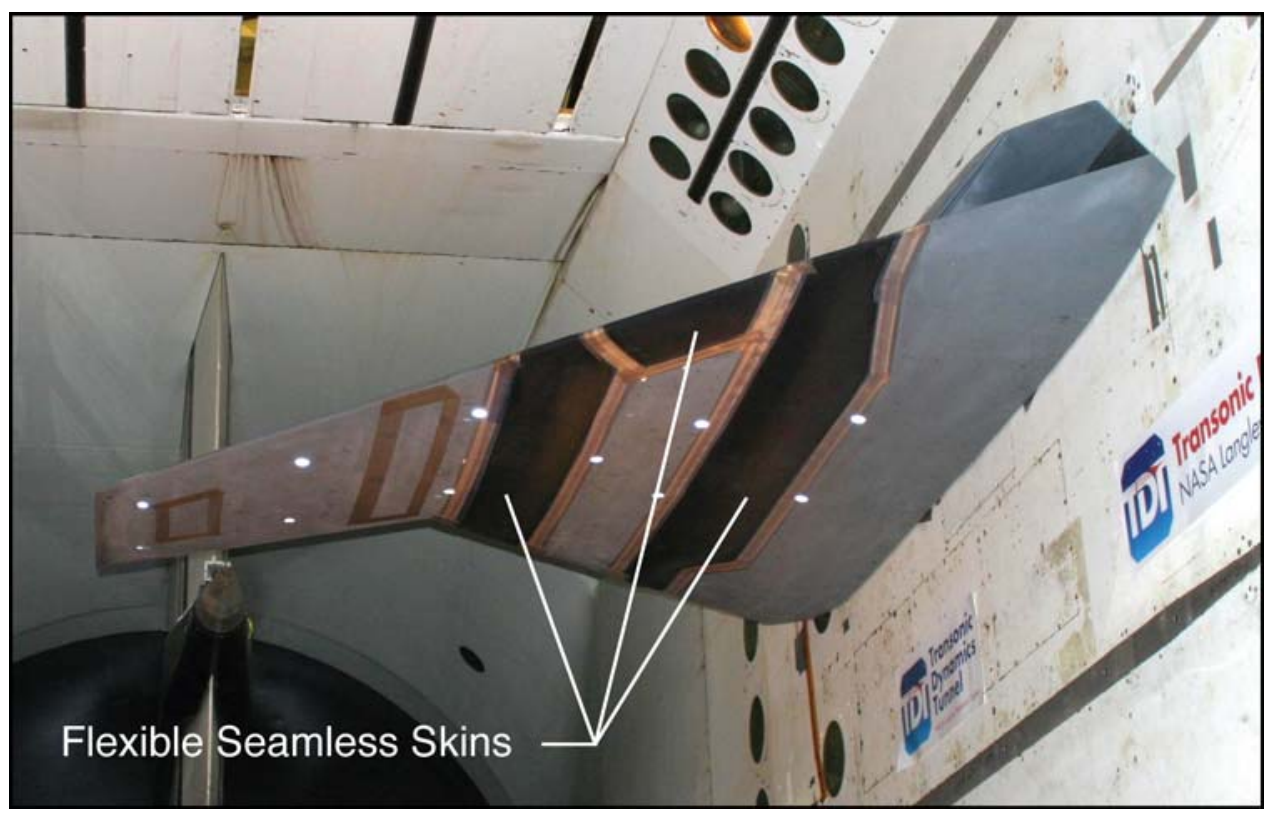

Figure 9. Lockheed Martin MAS Model Mounted in TDT

During testing at 0.8 Mach number and 45 degrees wing fold angle, the first condition exhibiting skin dynamics was encountered. This incident has direct application to any full-scale flight vehicle that would employ this type of design and reveals the importance of understanding and being able to predict the dynamics of the flexible skin used in the wing fold regions of the model. At this test condition the following events occurred in rapid sequence: sealant tape that bonds the inboard flexible skin to the fuselage was seen to depart the wind tunnel model; immediately following tape departure, traveling waves developed in the flexible skin; shortly after the establishment of traveling waves, the model experienced flutter that led test engineers to rapidly terminate testing.

Subsequent data examination revealed that following the departure of the sealant tape, the pressure in the wing cavity beneath the flexible skin dropped to about the level of the suction pressure on the wing upper surface, thereby inciting the traveling wave. Also, the frequency of the sustained traveling wave is approximately half that of the flutter mode. It is not believed that the oscillation of the flexible skin caused the model to flutter since the revised flutter analysis reveals the existence of the second mode instability at this condition. However, it is believed that the motion of the flexible skin may have helped trigger the flutter incident.

Figure 10 contains a plot of the unsteady pressure data from a high response total pressure transducer located on the upper surface near the inboard skin leading edge. The unsteady values plotted in figure 10 are the measured total pressure data with the free stream static pressure value subtracted. Also, the flexible skin cavity pressure is plotted as measured by a steady pressure system referenced to free stream static.

One can see a relatively constant cavity pressure at the beginning of the data record. The cavity pressure then undergoes an excursion, corresponding to a loss of sealant tape, followed by a rise in cavity pressure. As the cavity pressure rises to approximately $-0.45 \mathrm{psi}$, the first traveling wave of the skin is observed. As the wave passes the unsteady pressure transducer, a pressure impulse is recorded from what is believed to be a local shock wave moving with the traveling wave on the flexible skin. As the cavity pressure continues to rise, the traveling waves become more frequent as recorded by additional impulses in the unsteady pressure measurement. At approximately 56 seconds, the traveling waves become regular in frequency followed by model flutter onset at approximately 58 seconds. The sharp rise in pressure seen at approximately 60 seconds is the result of an emergency reduction of flow velocity, referred to as a "tunnel bypass." Finally, one can also notice that the cavity pressure values fluctuate erratically during the traveling wave occurrences. Pressure fluctuations within the cavity would be expected due to the changes in cavity volume caused by the lifting and falling of the flexible skin. The cavity pressures presented in figure 10 were measured with a steady pressure system that requires settling time for accurate measurements. Thus, while the trends are believed to be captured, the values are not accurate. 


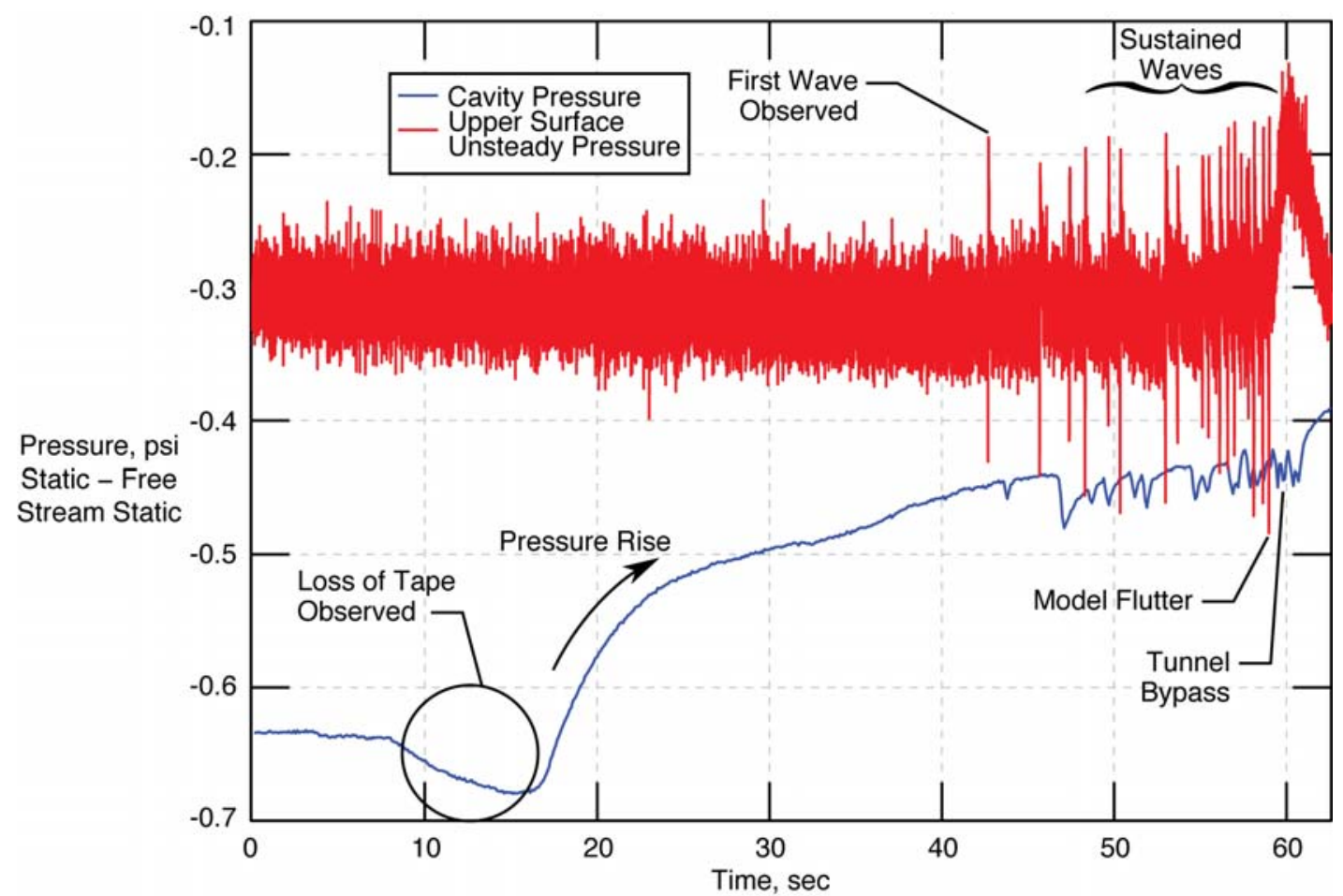

Figure 10. Pressure Data Acquired During Sustained Traveling Waves, 45 deg Wing Fold.

The observed motion of the flexible skin exhibits some of the characteristics of traveling wave panel flutter that can occur during transonic speeds. ${ }^{5}$ One such characteristic is the speed of the traveling wave. Figure 11 is a plot of data from two high response unsteady pressure transducers located on the upper surface near the inboard skin. This data record was acquired during sustained traveling wave oscillations at 0.8 Mach number and the time sequence is a subset of the data presented in figure 10. Physical separation between the upstream and downstream pressure transducers was 56.29 inches. Temporal separation between pulses observed by each transducer was a consistent 0.193 seconds. Combining the physical and temporal separations revealed a traveling wave speed of 292 inches per second (24.3 feet per second). If one employs the equations for predicting traveling wave speed contained in reference 5 and uses rough estimates for the density and tension of the flexible skins, the resulting speed is in reasonable agreement with 24.3 feet per second. This result adds confidence to the suspicion that this observed traveling wave was indeed panel flutter. 


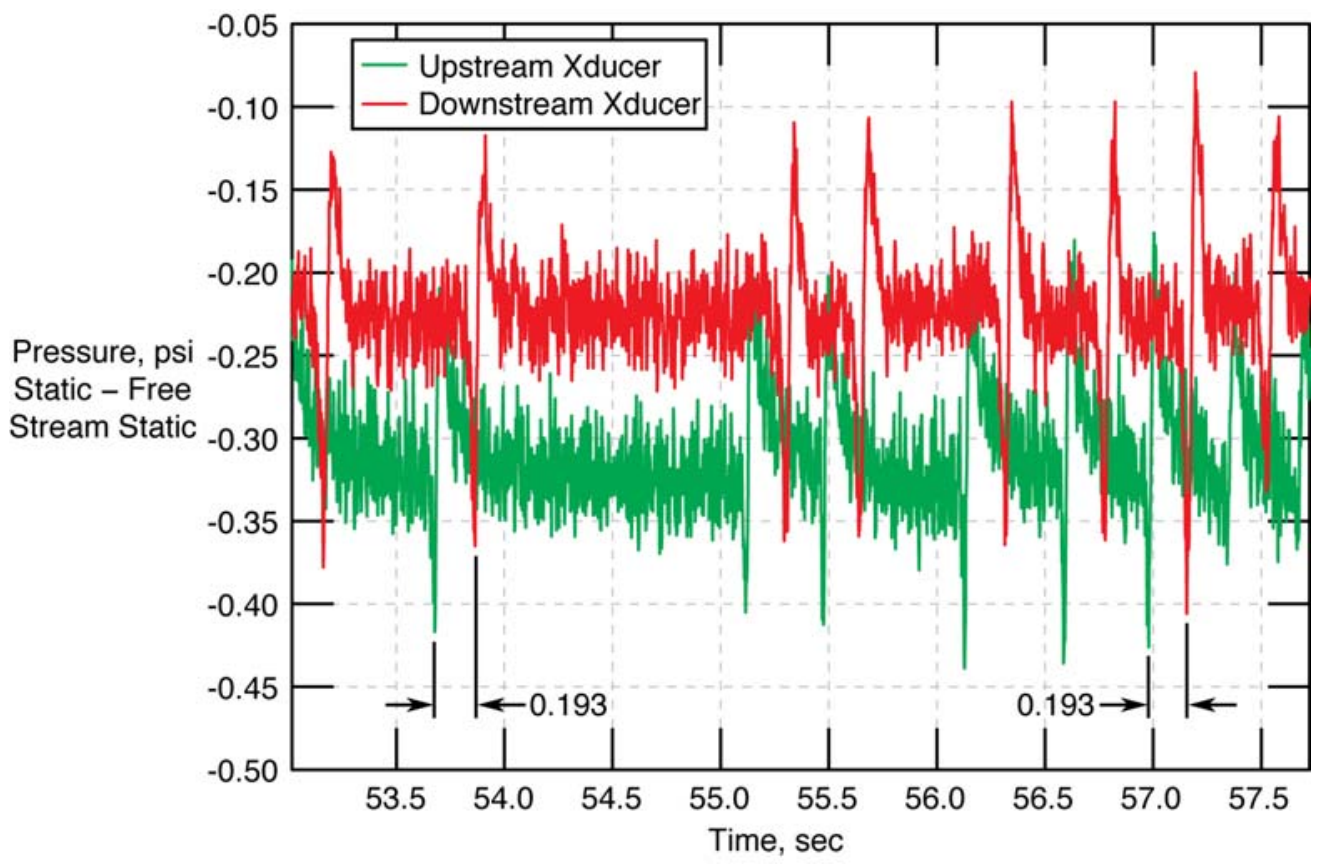

Figure 11. Unsteady Pressures Near Inner Wing Fold Line, Mach 0.8, 45 deg Wing Fold, During Sustained Traveling Wave Motion.

A similar sequence of events, vacuum pressure loss - traveling wave motion of the skin - model dynamics, also occurred at a Mach number of 0.9. In this second event, the model angle of attack was at 4.5 degrees and the occurrence was sensitive to model angle of attack. The model dynamic motion observed during this second occurrence was more representative of a limit cycle oscillation rather than a divergent flutter oscillation.

Finally, a different type of skin motion was observed, representative of a standing wave, on the outboard skin near the leading edge at 0.8 Mach number and angles of attack in the vicinity of 9 degrees. In this third encounter, the tunnel dynamic pressure was low and no significant vibrations of the model were measured. Once again the skin vacuum pressure was at a reduced value due to system leakages that were later corrected.

Since these three observed occurrences were rare and precipitated by vacuum system leakages or improper pressures, it was determined that the flexible skin successfully met criterion 4.

\section{E. Success Criterion 5. Demonstrate successful use of flight representative hardware to operate the actuation systems.}

Detailed in references 1 and 3 is the structural design of the model incorporating realistic flight hardware. An example of this is the use of F-16 leading edge flap actuators to carry the loads through the hinge lines and operate the wing fold motion. Additionally, the vehicle skin and spar design mimicked that of a semi-monocoque flight vehicle including critically loaded composite parts. All components performed well allowing the model to be tested to the maximum anticipated loads.

\section{F. Success Criterion 6: Demonstrate successful mating of the morphing structures and seamless gap closures.}

This criterion was developed as a result of lessons learned from the swing-wing wind tunnel tests conducted during the development of the F-111 and the F-14. ${ }^{6}$ The intent was to demonstrate the ability of the wind tunnel model to obtain a seamless mating of the moving wing surface and the fuselage. To address this criterion, Lockheed Martin designed the leading edge flap with the purpose of closing the gap between the inboard wing and fuselage when the model was in the dash configuration. However, the elastic deflection of the wing under aerodynamic load was not known prior to testing. Therefore, the motion of the wing and leading edge flap was intentionally stopped short of making contact with the fuselage. While the incorporation of this flap was a novel approach to this requirement, a tighter tolerance in closing the gap would be required in order to fully meet this criterion. 


\section{G. Success Criterion 7: Acquire specified sets of wind tunnel data to correlate with design analysis.}

A multitude of wind tunnel data was acquired including balance data, steady and unsteady pressure data, structural loads, and accelerations. Lockheed Martin conducted detailed comparisons of the wind tunnel data with CFD analyses and finite element model structural load predictions. Comparisons of the wind tunnel data with the Lockheed Martin predictions are presented in reference 1.

\section{Lessons Learned}

In an effort to aid future wind tunnel tests of morphing aircraft, several lessons learned during this wind tunnel test are documented below.

\section{A. Support Strut Stiffness}

The LM MAS wind tunnel model was the heaviest sidewall-mounted model ever tested in the forty-seven year history of the TDT. Upon model installation, the support strut was found to be significantly more flexible in pitch than anticipated. This unexpected loss of stiffness lowered the flutter dynamic pressure of the model to within the desired wind tunnel test envelope. A better understanding of the wind tunnel support strut stiffness would have prevented the unexpected flutter encounters. Experimental data now exists documenting the current stiffness of the TDT sidewall strut. Future wind tunnel tests of large sidewall-mounted models need to consider the support strut stiffness in order to properly understand possible coupling of model and support system dynamics.

After post-test inspection of the strut assembly and further analysis, the difference in expected vs. actual pitch stiffness can be attributed to the following:

1) The pitch stiffness of the strut is less than anticipated due to a calculation error committed by NASA. This error accounts for about $5 \%$ of the loss in stiffness.

2) Undersized bolts were discovered on the pitch drive mechanism. These bolts would loosen with changes in AOA causing a loss in stiffness and introducing free play. Incorrect shoulder bolts were also discovered on a connecting arm that failed to engage the bolt shoulders across the pitch drive mechanism. The incorrect bolts account for approximately $35 \%$ of the loss in stiffness.

3) The pitch drive system attachment, electric motor drive system, and bearings are difficult to analytically model and consist of large components. Due to the size of these components, they were originally modeled as rigid connections to the support strut. Calculations were done of the drive system jackscrew connection and other hardware components which predicted high stiffness. The source of this additional flexibility is not entirely understood. Experimental results, however, indicate that the remainder of the loss in stiffness is attributed to these components.

Following the Lockheed Martin MAS test, correct bolts were installed (item 2) on the support strut system that increased its stiffness and eliminated free play. NASA conducted new flutter analyses with this increased pitch stiffness by making slight modifications to the boundary conditions in the NASTRAN finite element model. The analysis shows that the flutter boundary is extremely sensitive to pitch stiffness, and results indicate that the test could now be run without encountering flutter within the desired test envelope.

\section{B. Lowered Flutter Dynamic Pressure at Moderate Fold Angles}

The LM MAS wind tunnel model accomplished morphing by a novel mechanization of out-of-plane wing folding. Through analysis and test, the flutter dynamic pressure was found to be a strong function of wing fold angle. It was learned through post-test flutter analyses that the critical flutter mode changes as fold angle varies. For fold angles between approximately zero and 20 degrees, and between 80 and 130 degrees the flutter mode is characterized by a full-body pitch motion about the balance and supporting strut. However, fold angles between approximately 20 and 80 degrees, the critical flutter mode is characterized by an in-plane motion of the outboard wing coupled with a torsion motion of the inboard wing and a full-body pitch/yaw motion about the balance and supporting strut. Additionally, ongoing research at NASA indicates that a coupling between in-plane motion and pitch can induce flutter at a lower dynamic pressure when there is a vertical offset in center of gravity. Moderate wing fold angles in the vicinity of 45 degrees can aggravate such a coupling.

The lesson learned is that close attention must be paid to this mode of vibration during flutter analyses with moderate fold angles. The small damping magnitude combined with the instability only existing for a select range of velocities makes this an easy mode to overlook. LM is applying this lesson in the analysis of a similarly designed unmanned flight demonstrator aircraft. 


\section{Wing Fold Control}

As the drive motor power was turned off, brakes were engaged to hold wing position. As actuation system power was cycled off and on, a slight amount of brake slippage allowed a small drive train slippage undetected by the wing fold controller. Continuous power cycles would lead to a slight offset in desired vs. actual wing position over time. During the wind tunnel test, the wing position was re-zeroed at the beginning of each day and checked at the end of each wind-on period. Wing position was found to remain within acceptable levels for all test conditions, and position error was slightly larger than the threshold of detection after hours of continuous cycles. However, a flight vehicle should employ a method to retain the drive train position measurement without power applied in order to eliminate the need to re-zero wing position readings on a daily basis.

\section{Effects of Wing Fold Motion on Balance Data}

Typical TDT data acquisition system routines involve an angle of attack correction applied to balance loads. Adjustments in balance orientation change the internal loadings due to gravity, which must be subtracted to correctly obtain the externally applied aerodynamic loads. The LM morphing aircraft, however, creates an additional problem due to the shift in CG associated with wing motion. Therefore, a two-dimensional weight tare routine was developed by the TDT data system team to support this test. Balance check loads confirmed that the actual external load could be measured to within one percent accuracy in all components regardless of the angle of attack or wing fold position. Anticipation of this requirement and the experimental trials prior to wind tunnel entry made this development possible. Therefore, this requirement must also be anticipated for future morphing designs to permit proper weight-tare corrections.

\section{E. Seamless Skin Characteristics}

The seamless skins produced an unexpected amount of "stiction" with the underlying finger skin structure. This caused unpredictable and unusual readings on wing fold hinge moments during actuation. Additionally, this created a large amount of hysteresis since the stiction would stress the skin differently depending upon the direction of motion. This unusual loading required a complicated skin-tare data routine to determine the aerodynamic hinge moments from the total measured loads. Extensive post-test data reduction was required of the TDT data system team to extract the aerodynamic loads for each test condition.

Additionally, to maintain their shape the flexible skins required a vacuum system when exposed to aerodynamic loads. This system worked well throughout most of the test, however, loss of suction during some conditions resulted in significant skin dynamics. As a result of the wind tunnel test, the minimum required vacuum pressures were determined for the varying conditions.

\section{F. Motor Current Feedback Problem}

As discussed in the section detailing success criterion 1, the wing actuation system experienced a current feedback problem associated with morphing from dash to loiter configurations under $1 \mathrm{~g}$ load. The actuation system design did not anticipate the aerodynamic load acting in the direction of desired motion. As previously mentioned, a solution was identified but deemed unnecessary to accomplish the test objectives. Therefore, future actuation systems need to consider aerodynamic loads that act in the desired direction of wing morphing and not only the loads that oppose the wing morphing motion.

\section{Conclusion}

The Lockheed Martin Variant of the MAS phase II wind tunnel program successfully met all DARPA directed objectives. The validity of the morphing design was quantified by the wind tunnel test success criteria. The model was able to morph under a $1 \mathrm{~g}$ load and maintained a constant structural position under loads in excess of $1 \mathrm{~g}$. Wing fold position was accurately controlled and was predictable and repeatable lending confidence about the ability to control a full-span flight vehicle. Data presented here documents the loading encountered for varying wing fold angles at different angles of attack. Many structural components utilized realistic aircraft design features and actual components.

The data acquired during different morphing configurations can be used to study fuselage/wing interactions and dynamic response due to wing motion. Furthermore, the presented lessons learned and difficulties that were encountered will aid future wind tunnel programs of morphing vehicles.

No aeroelastic instabilities were encountered due to the wing actuation or morphing systems. However, due to the unexpected support strut flexibility, aeroelastic instabilities were observed. One occurrence in particular brought attention to a mode-switching characteristic that may impact the analysis of a future flight demonstrator. 
Additionally, after a vacuum failure the model encountered a flutter condition immediately following significant flexible skin dynamics. It cannot be concluded that the flexible skin dynamics played a role in the vehicle dynamics, however, the occurrence prior to a flutter onset is interesting to note. Finally, post-test modifications to the wind tunnel support strut have increased its pitch stiffness. Recent flutter analyses, that include the increased stiffness, predict that the LM MAS model could test within its entire desired envelope without experiencing flutter.

\section{Acknowledgments}

Many organizations contributed to the development and completion of the MAS wind tunnel test program. First of all, the Defense Sciences Office of DARPA created and organized this effort. Special recognition goes to Dr. Ephrahim Garcia whose original efforts led to the authorization for this project. Also the Air Vehicles Directorate, AFRL, was an active participant in the oversight of the model design requirements and liaison between DARPA and Lockheed Martin during the critical early stages of MAS development. Numerous Lockheed Martin design and test engineers, and sub-contractors, conceived and developed the morphing model. In particular we would like to recognize Derek Bye and Charles Chase as the Lockheed Martin program managers, and Advanced Technologies Incorporated for constructing the model. Finally, our thanks go to the skilled professional staff at the TDT facility and NASA support contractors who provided additional assistance at critical times, kept the test on schedule and removed the MAS model from the TDT in the same form as it entered.

\section{References}

${ }^{1}$ Love, M. H, Zink, S., Bye, D. R., Rizk, S. N., Stroud, R. L., and White, D. W., "Demonstration of Morphing Technology through Ground and Wind Tunnel Tests,” AIAA 2007-1729, May 2007.

${ }^{2}$ Weisshaar, T. A., “Morphing Aircraft Technology Progress, New Shapes for Aircraft Design,” Paper Submitted to AIAA for publication, 2007.

${ }^{3}$ Kehs, K. K.; Love, M. H., Box, D., "Model and Test Information Report, 1/2 Scale Morphing Aircraft Structures Model," FZT-672, Lockheed Martin, December 2005.

${ }^{4}$ Staff of the Aeroelasticity Branch, “The Langley Transonic Dynamics Tunnel,” Langley Working Paper LWP-799, September, 1969.

${ }^{5}$ Greenspon, J.E., and Goldman, R. L., "Flutter of Thin Panels at Subsonic and Supersonic Speeds,” The Martin Company. OSR Technical Report No. 57-65, ASTIA Document No. 136 560. July, 1957.

${ }^{6}$ Kress, R., “Variable Sweep Wing Design,” AIAA 80-3043, 1980. 